Case Report

\title{
Adrenal Histoplasmosis : A rare cause of bilateral Adrenomegaly
}

\author{
Subedi PP, Chapagain S, Thakur DK, Poudyal S, Luitel BR, Chalise PR, Sharma UK, Gyawali PR, Shrestha GK \\ Department of Surgery, Urology Unit, Tribhuvan University Teaching Hospital, Maharajgunj, Kathmandu, Nepal \\ Correspondence: Dr Pradip Prasad Subedi, MCh Resident, Email: subedipradip81@gmail.com
}

Abstract
Histoplasmosis is an opportunistic infection caused by a dimorphic fungus Histoplasma
capsulatum. Histoplasmosis causing bilateral adrenal involvement is rare and usually occurs only
in immunocompromised individuals with disseminated disease. Here we report a case of bilateral
adrenal histoplasmosis with disseminated disease in an immunocompetent patient.

\section{Introduction}

Histoplasmosis usually occurs in immunocompromised hosts. It is caused by the fungus Histoplasma capsulatum. The infection is acquired through inhalation of its spores. Spores are found in the soil contaminated with bird and bat droppings. The infection is usually subclinical and leads to transient pulmonary infection that resolves after a flulike symptoms. In immunocompromised hosts it can lead to disseminated disease that can involve the liver, lymph nodes, spleen, bone marrow and adrenal gland. ${ }^{1,2}$ Our patient is a middle aged man who presented with non specific symptoms of systemic disease who on investigation was found to have bilateral adrenal mass on imaging studies and adrenal histoplasmosis on open incisional biopsy after two inconclusive fine needle aspiration biopsy (FNAB). A brief review of epidemiology, microbiology, clinical features, imaging characteristics, FNAB findings and treatment of adrenal Histoplasmosis is presented in this report.

\section{Case report}

A 52 years old male presented with complains of low grade fever, fatigue and weight loss for 8 months. He had no known comorbidities. He was a past smoker. He looked ill though his vitals were stable and his systemic examination was normal. His routine investigations were normal except for a WBC count of $3600 / \mathrm{mm}^{3}$. His ultrasound of the abdomen showed heterogenous enlargement of bilateral adrenal glands. Contrast enhanced CT abdomen revealed a heterogeneously enhancing soft tissue density lesion with central hyperdensity in bilateral suprarenal areas suggestive of malignant mass with the differential of metastasis. (Figure 1) Metabolic evaluations (24 hour urinary VMA, urinary metanephrines and 24 hour urinary cortisols with respect to the adrenals were normal. Work up for tuberculosis and lymphoma and serological test for HIV was normal. Search for a possible primary tumor sites (chest $\mathrm{X}$-ray, ultrasound of thyroid gland that canmetastasizeto the adrenal revealed normal findings. USG guided fine needle aspiration biopsy FNAB from the adrenal tumor was taken but it was inconclusive. A repeat FNAB was done which too was inconclusive.By anterior subcostal incision, an open incisional biopsy from the left adrenal gland was taken under general anaesthesia and reports were awaited. The patient was stable during the first 20 hours after surgery when he developed hypothermia with temperature of $92 \mathrm{~F}$, low blood pressure of $80 / 50 \mathrm{~mm}$ of $\mathrm{Hg}$ and altered sensorium. The patient was jointly seen by the urology, endocrinology and anaesthesia team; but the cause of his sudden deterioration could be ascribed to sepsis. His investigations then were all normal except for a low WBC count of $2200 / \mathrm{mm}^{3}$. Blood culture was sent and antibiotics was upgraded to meropenemnd supportive management for hypothermia was begun. His organ systems were taken care of and steroid therapy was started with hydrocortisone $100 \mathrm{mg}$ IV thrice a day. For the ensuing week the patient continued to be hypothermic with minimum temperature record of $94 \mathrm{~F}$ and $\mathrm{WBC}$ count of $2100 / \mathrm{mm}^{3}$ though clinically stable. Reports of the biopsy revealed adrenal histoplasmosis. (Figure. 2) The patient was immediately started on Itraconazole $200 \mathrm{mg}$ BD per oral for 10 months. Hewas kept in the ward for 10 days and at discharge he had a temperature of $98 \mathrm{~F}$, WBC count of $3200 / \mathrm{mm}^{3}$ and an increased sense of well being. The patient is doing well and has normal complete blood count after one and half years of follow up. He is scheduled to undergo CT scan of the abdomen at 2 years of follow up as there is higher chance of recurrence. 


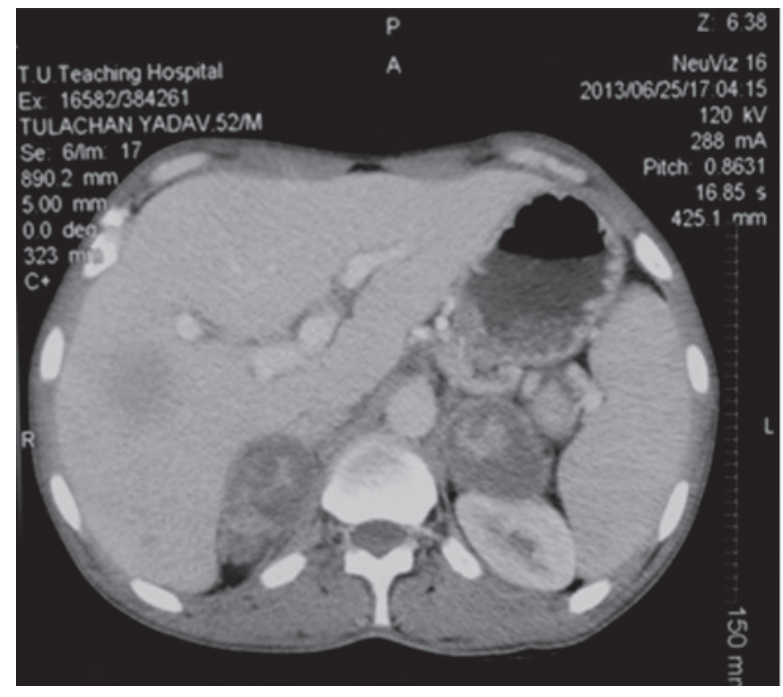

Figure 1: Heterogenous enhancing bilateral adrenal masses

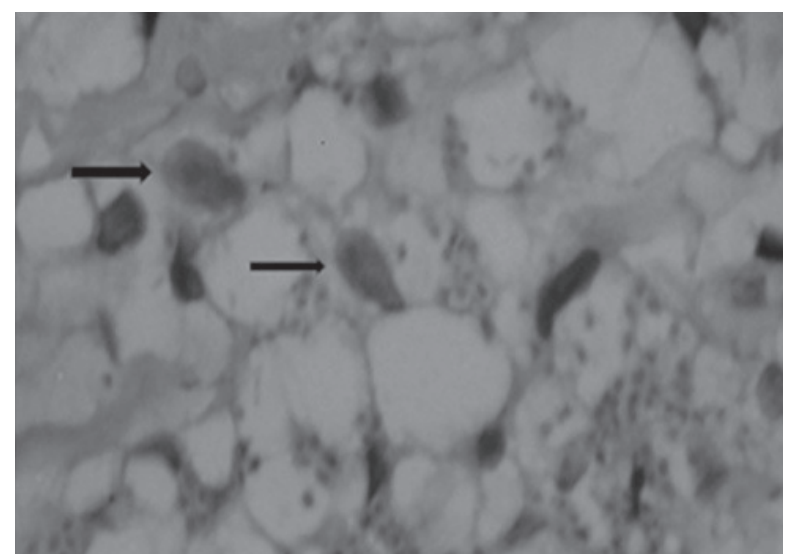

Figure 2: Yeast cells seen in the adrenal biopsy specimen

\section{Discussion}

Disseminated histoplasmosis is a potentially fatal disease caused by dimorphic fungus Histoplasma capsulatum. ${ }^{3}$ Histoplasma capsulatum are found in soil contaminated with bird and bat droppings. Infection is acquired by inhalation of spores. ${ }^{2}$ The fungus causes transient pulmonary infection without sequalae in immunocompetent host but can cause chronic pulmonary infection and life threatening disseminated disease in immunocompromised hosts. ${ }^{4}$ Males are more commonly infected. In disseminated disease liver, spleen, lymph nodes, bone marrow and adrenals are frequently involved. In the adrenals unilateral involvement is more common. Bilateral involvement of the adrenals is rare and so is the disseminated disease.
Disseminated disease present with pyrexia, anorexia, nausea, vomiting, fatigue and weight loss as in our case. These are non specific and often presents in malignancy and other common infections. ${ }^{5}$ The characteristic CT finding is a symmetrical bilateral adrenal enlargement with central hypodensity. However these findings can be found in adrenal haemorrhage, lymphoma, metastasis, tuberculosis, cryptococcosis, blastomycosis or aspergilosis. However findings of rim and septal enhancement would narrow the diagnosis to histoplasmosis and tuberculosis. ${ }^{6}$ So the need for a biopsy is important. FNAB shows cluster of macrophages with abundant cytoplasm containing the yeast. The yeast can also be seen extracellularly in the smears when the fragile cytoplasm of macrophages extrude out. $^{2}$ The disease is treated with intravenous amphoterecin $\mathrm{B}$ or oral itraconazole depending on the clinical status of the patient. ${ }^{7}$ In our case as well we thought of starting amphotericin B, but due to bad side effects and logistic issues, we have started Itraconazole, to which the patient responded well.

High index of suspicion is required to diagnose disseminated histoplasmosis in a list of differentials when imaging is inconclusive.

\section{References}

1. Mukherjee, J. J., Villa, M. L., Tan, L. \& Lee, K. O. Bilateral adrenal masses due to histoplasmosis. J. Clin. Endocrinol. Metab.90, 6725-6726 (2005).

2. Wahab, N. A., Mohd, R., Zainudin, S. \& Kamaruddin, N. A. Case report: Adrenal involvement in histoplasmosis. EXCLI Journal 12, 1-4 (2013).

3. Dwivedi, M., Piparsania, B., Issar, P. \& Dewangan, L. Disseminated histoplasmosis of adrenal gland. Indian J. Radiol. Imaging16, 651 (2006).

4. Larbcharoensub, N. et al. Adrenal histoplasmosis : a case series and review of literature. Southeast Asian J Trop Med Public Health 42, (2011).

5. Rana, C., Krishnani, N. \& Kumari, N. Bilateral adrenal histoplasmosis in immunocompetent patients. Diagn. Cytopathol.39, 294-6 (2011).

6. Grover, S. B., Midha, N., Gupta, M., Sharma, U. \& Talib, V. H. Imaging spectrum in disseminated histoplasmosis: case report and brief review. Australas. Radiol.49, 175-8 (2005).

7. Wheat, L. J. et al. Clinical practice guidelines for the management of patients with histoplasmosis: 2007 update by the Infectious Diseases Society of America. Clin. Infect. Dis.45, 807-825 (2007). 